\title{
Novel technique to prevent central airway blood flooding during transbronchial cryobiopsy
}

\author{
Masahide Oki, Hideo Saka \\ Department of Respiratory Medicine, Nagoya Medical Center, Nagoya, Japan \\ Correspondence to: Masahide Oki, MD. Department of Respiratory Medicine, Nagoya Medical Center, 4-1-1 Sannomaru, Naka-ku, Nagoya 460-0001, \\ Japan. Email: masahideo@aol.com.
}

Submitted Feb 01, 2019. Accepted for publication Apr 11, 2019.

doi: $10.21037 /$ jtd.2019.04.59

View this article at: http://dx.doi.org/10.21037/jtd.2019.04.59

Transbronchial cryobiopsy is useful for diagnosing diffuse parenchymal lung diseases, as well as localized lung lesions (1-5). It provides larger and higher quality specimens without crush artifacts compared to those obtained by conventional biopsy forceps (6). As transbronchial cryobiopsy samples tend to be larger, the risk of bleeding from the biopsy site is higher than that associated with forceps biopsy. This occasionally causes moderate or severe bleeding, so a bronchial balloon blocker has been prophylactically used to prevent flooding of blood into the central airway $(7,8)$. Although the balloon occlusion method is effective to avoid severe bleeding (8-11), guiding the balloon blocker to the occlusion site is sometimes challenging and complicates the cryobiopsy procedure. To facilitate the cryobiopsy procedure for parenchymal lung lesions, we developed a simpler technique to prevent bleeding by wedging the tip of a long, small-diameter silicone endobronchial tube into the target bronchus transnasally under bronchoscopic control. We herein report this technique.

\section{Case 1}

An 86-year-old man with a history of prostate carcinoma and myelodysplastic syndrome was referred to our hospital due to a chest $\mathrm{X}$-ray abnormality. His chest $\mathrm{X}$-ray and computed tomography scan revealed a localized lesion measuring $37 \mathrm{~mm}$ in the longest diameter in the right upper lobe lung. Bronchoscopy was performed under local anesthesia and conscious sedation with midazolam and fentanyl. A sterilized, customized $5 \mathrm{~mm}$ inner diameter silicone tube (Phycon tube, SH No.5; Fuji Systems, Tokyo,
Japan; Figure $1 A, B$ ) was used. The silicone tube was cut to a length of $40 \mathrm{~cm}$, and the distal tip was cut diagonally. A standard connector from a $5 \mathrm{~mm}$ endotracheal tube was attached to the proximal end of the tube to facilitate grasping. The tube was advanced through the nose into the trachea under bronchoscopic control using a $4.0 \mathrm{~mm}$ thin bronchoscope (BF-P260F; Olympus, Tokyo, Japan; Figure $1 B$ ). After locally anesthetizing the bronchus with lidocaine, the tube was further advanced and firmly wedged into the posterior segment of the right upper lobe (right B2) (Figure $2 A, B, C$ ). Then, a thin bronchoscope was advanced into the right $\mathrm{B} 2 \mathrm{~b}$, and a radial endobronchial ultrasound probe was advanced toward the target lesion under fluoroscopy. After localizing the target lesion by endobronchial ultrasound, a forceps biopsy was performed to obtain eight specimens. Then the location of the tube was bronchoscopically reconfirmed, and a transbronchial cryobiopsy was performed using a $1.9 \mathrm{~mm}$ cryoprobe (ERBE, Tubingen, Germany). Bleeding was minimal (Figure 2D) and there were no other complications. The specimens obtained by forceps biopsy and cryobiopsy contained adenocarcinomas.

\section{Case 2}

A 58-year-old man without a medical history underwent a medical examination. A chest $\mathrm{X}$-ray revealed diffuse reticular opacities, mainly in the bilateral middle and lower lung lobes. He was referred to our hospital for an examination during which he underwent bronchoscopy under conscious sedation. First, we attempted nasobronchial intubation with insertion of the $40 \mathrm{~cm}$ tube into the 

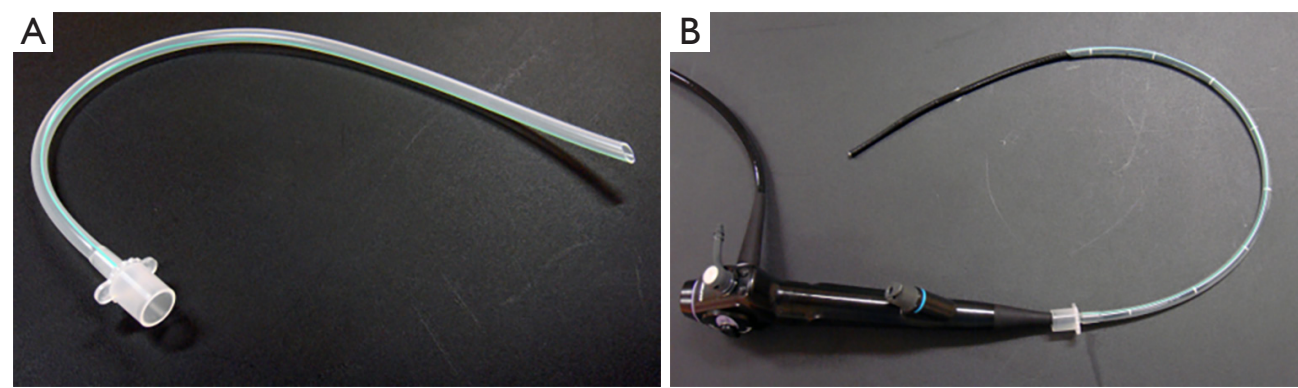

Figure 1 Instruments for the tube-wedging method. (A) A customized 5.0-mm silicone endobronchial tube with a connecter; (B) the tube attached to a $4.0 \mathrm{~mm}$ bronchoscope.
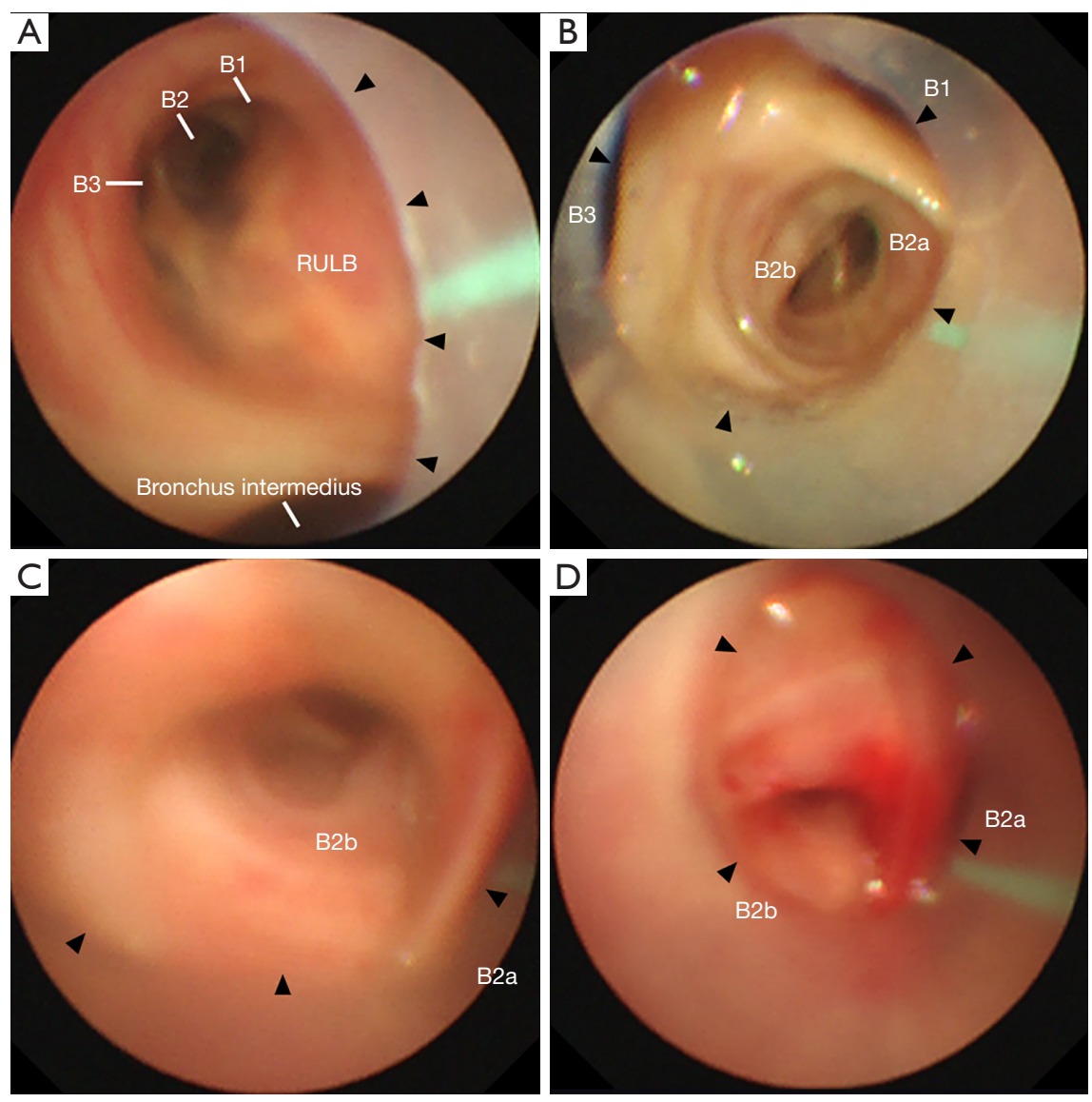

Figure 2 A silicone tube (arrowheads) was advanced through the right upper lobe bronchus (RULB) (A), and then through the right B2 (B); finally, it was wedged into the right B2 facing the bifurcation of B2a and B2b (C) under bronchoscopic control. Bronchoscopic view after the forceps biopsy and cryobiopsy shows no bleeding and no dislodgement of the silicone tube (D).

anterior basal segmental bronchus in the right lower lobe (right B8), but the tube was not long enough. Thus, after four specimens were obtained in the right B8 using biopsy forceps, we replaced the $40 \mathrm{~cm}$ tube with a $48 \mathrm{~cm}$ tube. The tube was advanced and wedged into the subsubsegmental bronchus (right B8bi) under bronchoscopy, and two biopsies were taken with a $1.9 \mathrm{~mm}$ cryoprobe from the lung parenchyma, followed by the right B8ai (Figure $3 A, B, C, D$ ) and right $\mathrm{B} 4 \mathrm{a}$; thus, a total of six cryobiopsy specimens were obtained. During the cryobiopsy, fluoroscopy showed an 

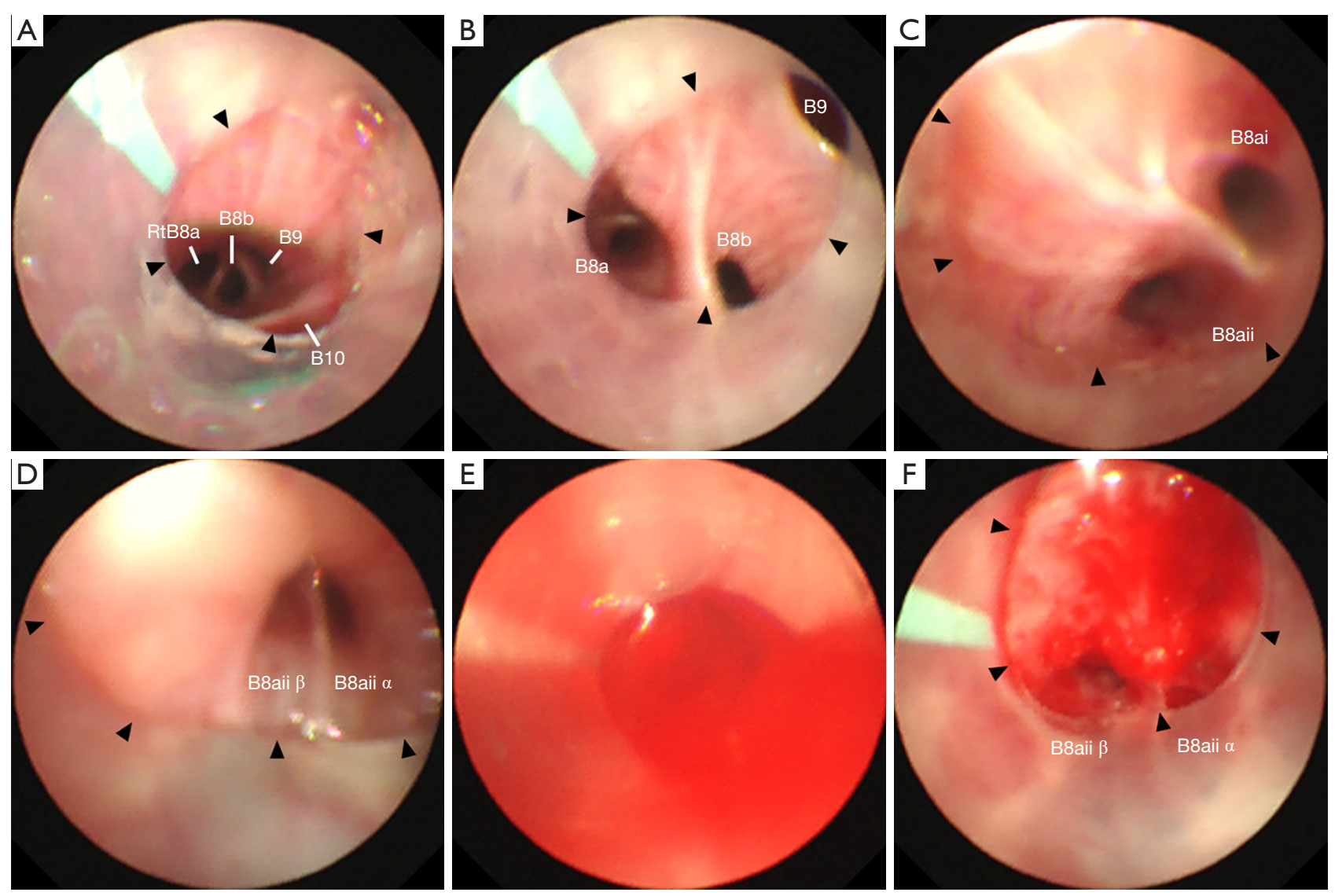

Figure 3 A silicone tube (arrowheads) was advanced under bronchoscopic control through the right basal bronchus (A), then through the right B8 (B), and then through the B8a (C); finally, it was wedged into the right B8aii, in which the next bronchi (B8aii $\alpha$, B8aii $\beta$ ) was seen (D). Bleeding occurred within the tube $(\mathrm{E})$. Bleeding was readily controlled by bronchoscopic suctioning $(\mathrm{F})$.

expanded opaque shadow, suggesting bleeding around the biopsy site, and blood flooded into the tube (Figure 3E). However, we were able to control the bleeding easily with bronchoscopic suctioning (Figure $3 F$ ). No blood flooded from the wedged tube into the central airway. The forceps biopsy specimen findings were non-diagnostic, while the cryobiopsy specimens showed pathological findings of suspected nonspecific interstitial pneumonia as well as silicosis. Subsequently, he was diagnosed with Sjögren's syndrome and received steroid therapy.

The results of our initial 10 cases with transbronchial cryobiopsy using the "tube-wedging method" under conscious sedation are shown in Table S1. Where bleeding occurred, it was limited to within the tube in all patients. Bleeding was graded as "no bleeding" or "mild bleeding" in all cases ["mild bleeding" requires suction to clear but no other bronchoscopic procedure, as classified by Hetzel et al. (10)]. This study was approved by the Institutional Review Board of Nagoya Medical Center (identifier: 2018-068).

Bleeding and pneumothorax are major complications associated with transbronchial cryobiopsy of parenchymal lung lesions $(10,11)$. The reported frequency of bleeding complications varies widely in the literature, even in meta-analyses, ranging from $4.9 \%$ to $39 \%$ (1-3). Most cases involve mild or moderate bleeding, although severe bleeding causing a life-threatening condition has been reported occasionally (12). In cases with blood flooding into the central airway, coughing is induced; this causes further bleeding, which makes bronchoscopic control difficult. Thus, a prophylactic technique for protecting the nonbleeding lung area is necessary. Although several techniques, such as balloon blockers $(7,13,14)$ and sequential use of two scopes (15), have been proposed, the prophylactic placement of a balloon blocker, which reduces the frequency of moderate or severe bleeding (8-11), seems to be the most effective; thus, its use during transbronchial cryobiopsy 
has been recommended $(2,10,11)$. However, the balloon occlusion technique is complex. Although several balloon occlusion techniques [e.g., use of a Fogarty balloon through the side channel of a double lumen tracheal tube $(7,11)$, an angioplasty balloon through a large tracheal tube (13), and an endobronchial blocker (14)] have been proposed, none of them seem to be optimal.

Our "tube-wedging method" has several advantages. By directly bridging into the leading bronchus, the established airway facilitates repeated insertion and removal of a thin bronchoscope. The wedging tube prevents central airway blood leakage by draining blood within the tube. In all our cases, bleeding was readily controlled bronchoscopically. Selective intubation into the segmental or deeper bronchus under bronchoscopic control is easily accomplished, except that into the apical segmental bronchus of the upper lung lobe. Displacement of the wedged tube occurred in one case, but holding the tube firmly prevents this from occurring. Another advantage of the tube-wedging method is the use of a small-diameter silicone tube, which allows a transnasal approach, and seems to be more comfortable for patients than the orotracheal approach using a larger tracheal tube. In fact, all patients tolerated the cryobiopsy procedures well under moderate conscious sedation with midazolam and fentanyl. The small-diameter tube allows the use of 4.0 or $4.2 \mathrm{~mm}$ thin bronchoscopes, which are particularly useful for peripheral localized pulmonary lesions $(16,17)$. By contrast, the lack of availability of therapeutic bronchoscopes with a large working channel and a $2.4 \mathrm{~mm}$ cryoprobe, the lack of availability of deep sedation or general anesthesia, and the inaccessibility of the apical segmental bronchus are limitations of the present technique.

In our early experience, the bleeding could easily be controlled in all cases by bronchoscopic suctioning alone. If the bleeding cannot be controlled by simple suction, airway blocking by wedging the bronchoscope or using a balloon blocker through the bronchoscope working channel would be the technique of choice. We have yet to encounter patients with moderate/severe bleeding; however, we are always prepared to manage unexpected massive bleedings. At our institution, we are always able to use therapeutic bronchoscopes with a large working channel, large endotracheal tubes, balloon blockers, and adrenaline for endobronchial administration.

In conclusion, our early experience suggests that the tube-wedging method is simple and safe, and may be a promising alternative to the balloon occlusion method for transbronchial cryobiopsies. Further studies are needed to confirm the usefulness of this technique.

\section{Acknowledgments}

None.

\section{Footnote}

Conflicts of Interest: The authors have no conflicts of interest to declare.

Ethical Statement: This study, including our initial 10 cases, was approved by the Institutional Review Board of Nagoya Medical Center (identifier: 2018-068). Informed consent for publication was obtained in writing from Cases 1 and 2 .

\section{References}

1. Johannson KA, Marcoux VS, Ronksley PE, et al. Diagnostic yield and complications of transbronchial lung cryobiopsy for interstitial lung disease: A systematic review and metaanalysis. Ann Am Thorac Soc 2016;13:1828-38.

2. Ravaglia C, Bonifazi M, Wells AU, et al. Safety and diagnostic yield of transbronchial lung cryobiopsy in diffuse parenchymal lung diseases: A comparative study versus video-assisted thoracoscopic lung biopsy and a systematic review of the literature. Respiration 2016;91:215-27.

3. Iftikhar IH, Alghothani L, Sardi A, et al. Transbronchial lung cryobiopsy and video-assisted thoracoscopic lung biopsy in the diagnosis of diffuse parenchymal lung disease: A meta-analysis of diagnostic test accuracy. Ann Am Thorac Soc 2017;14:1197-211.

4. Schuhmann M, Bostanci K, Bugalho A, et al. Endobronchial ultrasound-guided cryobiopsies in peripheral pulmonary lesions: A feasibility study. Eur Respir J 2014;43:233-9.

5. Sánchez-Cabral O, Martínez-Mendoza D, FernandezBussy S, et al. Utility of transbronchial lung cryobiopsy in non-interstitial diseases. Respiration 2017;94:285-92.

6. Schumann C, Hetzel J, Babiak AJ, et al. Cryoprobe biopsy increases the diagnostic yield in endobronchial tumor lesions. J Thorac Cardiovasc Surg 2010;140:417-21.

7. Hernández-González F, Lucena CM, Ramírez J, et al. Cryobiopsy in the diagnosis of diffuse interstitial lung disease: Yield and cost-effectiveness analysis. Arch Bronconeumol 2015;51:261-7. 
8. Dhooria S, Mehta RM, Srinivasan A, et al. The safety and efficacy of different methods for obtaining transbronchial lung cryobiopsy in diffuse lung diseases. Clin Respir J 2018;12:1711-20.

9. Poletti V, Hetzel J. Transbronchial cryobiopsy in diffuse parenchymal lung disease: Need for procedural standardization. Respiration 2015;90:275-8.

10. Hetzel J, Maldonado F, Ravaglia C, et al. Transbronchial cryobiopsies for the diagnosis of diffuse parenchymal lung diseases: Expert statement from the cryobiopsy working group on safety and utility and a call for standardization of the procedure. Respiration 2018;95:188-200.

11. Colella S, Haentschel M, Shah P, et al. Transbronchial lung cryobiopsy in Interstitial lung diseases: Best practice. Respiration 2018;95:383-91.

12. DiBardino DM, Haas AR, Lanfranco AR, et al. High complication rate after introduction of transbronchial cryobiopsy into clinical practice at an academic medical center. Ann Am Thorac Soc 2017;14:851-7.

13. Echevarria-Uraga JJ, Pérez-Izquierdo J, García-Garai

Cite this article as: Oki M, Saka H. Novel technique to prevent central airway blood flooding during transbronchial cryobiopsy. J Thorac Dis 2019;11(9):4085-4089. doi: 10.21037/ jtd.2019.04.59
$\mathrm{N}$, et al. Usefulness of an angioplasty balloon as selective bronchial blockade device after transbronchial cryobiopsy. Respirology 2016;21:1094-9.

14. Hohberger LA, DePew ZS, Utz JP, et al. Utilizing an endobronchial blocker and a flexible bronchoscope for transbronchial cryobiopsies in diffuse parenchymal lung disease. Respiration 2014;88:521-2.

15. Sriprasart T, Aragaki A, Baughman R, et al. A single US center experience of transbronchial lung cryobiopsy for diagnosing interstitial lung disease with a 2-scope technique. J Bronchology Interv Pulmonol 2017;24:131-5.

16. Ishida T, Asano F, Yamazaki K, et al. Virtual Navigation in Japan Trial Group. Virtual bronchoscopic navigation combined with endobronchial ultrasound to diagnose small peripheral pulmonary lesions: A randomised trial. Thorax 2011;66:1072-7.

17. Oki M, Saka H, Ando M, et al. Ultrathin bronchoscopy with multimodal devices for peripheral pulmonary lesions: A randomized trial. Am J Respir Crit Care Med 2015;192:468-76. 
Supplementary

Table S1 Procedural details of the initial 10 cases with cryobiopsy using the tube-wedging method

\begin{tabular}{|c|c|c|c|c|c|c|c|c|c|c|c|c|c|c|}
\hline No. & $\begin{array}{l}\text { Age, } \\
\text { years }\end{array}$ & Sex & $\begin{array}{l}\text { Lesion type, } \\
\text { longest diameter }\end{array}$ & $\begin{array}{l}\text { Cryoprobe } \\
\text { size, mm }\end{array}$ & $\begin{array}{l}\text { Bronchoscope } \\
\text { size, mm }\end{array}$ & $\begin{array}{l}\text { Lobar location } \\
\text { for cryobiopsy }\end{array}$ & $\begin{array}{l}\text { Number } \\
\text { of forceps } \\
\text { biopsies }\end{array}$ & $\begin{array}{l}\text { Number of } \\
\text { cryobiopsies }\end{array}$ & Results of forceps biopsy & Results of cryobiopsy & $\begin{array}{l}\text { Bleeding } \\
\text { into the } \\
\text { tube }\end{array}$ & $\begin{array}{l}\text { Blood } \\
\text { leakage } \\
\text { from the } \\
\text { tube }\end{array}$ & $\begin{array}{l}\text { Dislodge of } \\
\text { tube during } \\
\text { procedure }\end{array}$ & $\begin{array}{c}\text { Total } \\
\text { procedure } \\
\text { time, min }\end{array}$ \\
\hline 1 & 86 & Male & Localized, 37 mm & 1.9 & 4.0 & Rt. S2 & 8 & 1 & Adenocarcinoma & Adenocarcinoma & - & - & - & 24 \\
\hline 2 & 58 & Male & Diffuse & 1.9 & 4.0 & Rt. S4, S8 & 4 & 6 & Non-specific findings & Suspicious of NSIP, silicosis & + & - & - & 42 \\
\hline 3 & 43 & Male & Localized, 14 mm & 1.9 & 4.2 & Rt. S2 & 10 & 1 & Non-specific findings & Adenocarcinoma & - & - & + & 28 \\
\hline 4 & 48 & Female & Diffuse & 1.9 & 4.0 & Rt. S10 & 4 & 4 & Non-specific findings & Organizing pneumonia & + & - & - & 31 \\
\hline 5 & 51 & Male & Localized, $35 \mathrm{~mm}$ & 1.9 & 4.0 & Lt. S4 & 8 & 3 & Necrosis & $\begin{array}{l}\text { Necrosis, positive on } \\
\text { Ziehl-Neelsen stain }\end{array}$ & - & - & - & 23 \\
\hline 6 & 72 & Female & Localized, 19 mm & 1.9 & 4.0 & Rt. S9 & 8 & 1 & Adenocarcinoma & Non-specific findings & + & - & - & 43 \\
\hline 7 & 80 & Male & Localized, $51 \mathrm{~mm}$ & 1.9 & 4.2 & Rt. S2 & 7 & 3 & LCNEC & LCNEC & + & - & - & 28 \\
\hline 8 & 70 & Female & Localized, 23 mm & 1.9 & 4.0 & Rt. S8 & 8 & 2 & Non-specific findings & Non-specific findings & + & - & - & 34 \\
\hline 9 & 70 & Female & Localized, 31 mm & 1.9 & 4.2 & Rt. S8 & 5 & 2 & Adenocarcinoma & Adenocarcinoma & + & - & - & 33 \\
\hline 10 & 62 & Male & Diffuse & 1.9 & 4.2 & Lt. S10 & 9 & 4 & Organizing pneumonia & Organizing pneumonia & + & - & - & 50 \\
\hline
\end{tabular}

NSIP, non-specific interstitial pneumonia; LCNEC, large cell neuroendocrine carcinoma 